Reprinted from Archives of Environmental Health, published by HELDREF PUBLICATIONS, 4000 Albemarle Street, N.W., Washington, D.C. 20016.

\title{
Neurobehavioral Dysfunction in Firemen Exposed to Polychlorinated Biphenyls (PCBs): Possible Improvement after Detoxification
}

\author{
KAYE H. KILBURN, M.D. \\ University of Southern California \\ School of Medicine \\ Environmental Sciences Laboratory \\ 2025 Zonal Avenue \\ Los Angeles, California \\ RAPHAEL H. WARSAW \\ Workers' Disease Detection Services \\ 441 West Allen Avenue \\ San Dimas, California \\ MEGAN G. SHIELDS, M.D. \\ Foundation for Advancements in \\ Science and Education \\ 4801 Wilshire Boulevard \\ Los Angeles, California
}

\begin{abstract}
Fourteen firemen exposed to polychlorinated biphenyls (PCBs) and their byproducts generated in a transformer fire and explosion had neurophysiological and neuropsychological tests 6 mo after the fire. They were re-studied 6 wk later after undergoing 2-3 wk of an experimental detoxification program consisting of medically supervised diet, exercise, and sauna. A case-control comparison with firemen matched from the same department, but who did not participate in controlling the transformer fire, had shown significant impairment of memory for stories, visual images, and digits backwards. Cognitive function was impaired for block design, identifying embedded figures, and design association and recognition using Culture Fair. Making of trails and choice reaction time, which measured cognitive function and perceptual motor speed, were also impaired. These signs of protracted neurobehavioral impairment were attributed to PCBs and heat-produced byproducts. No relationship, however, was found between the firemen's serum or fat levels of PCBs as Arochlor 1248 and their type or degree of neurobehavioral impairment. Retesting following the detoxification program showed significantly improved scores on: three memory tests, block design, trails $B$, and embedded figures. Thus, there was significant reversibility of impairment after the detoxification interval. However self-appraisal scores for depression, anger, and fatigue-which were initially elevated-and for vigor-which was reduced-did not change across this interval.
\end{abstract}

TRICYCLIC polychlorinated aromatic compounds are formed in fires or explosions of electrical transformers or capacitors that contain polychlorinated biphenyls (PCBs) and benzenes. ${ }^{1-3}$ Thermolysis of PCBs at 500750 IC can generate polychlorinated dibenzofurans (PCDFS) and dibenzo- $p$-dioxins (PCDDS) in the laboratory, and they have also been recognized in samples from sites of fires and explosions. After inhalation of these chemicals in a Binghamton, New York, office building fire, the 
exposed population noted disorders of skin, eyes, and liver. Pain in the limbs and weakness have been observed after ingestion of PCBs. ${ }^{4}$ Also, chloracne, hirsuteness, blepharoconjunctivitis, hepatic dysfunction, and porphyria cutanea tarda have been observed after ingestion ${ }^{5}$ but not after inhalational exposures. ${ }^{6}$ A diminution or loss of peripheral sensory and motor function studies have been reported, accompanied by reduction of nerve conduction velocity. ${ }^{7,8}$ Except for nerve conduction velocity, neurobehavioral function studies have not been reported previously in human subjects with occupational or accidental exposure to PCBs.

Fourteen firemen were exposed to fumes, smoke, and gases from a transformer containing PCBs during a powerhouse fire on April 3, 1987. During the fire, a transformer containing PCBs exploded in a second floor transformer room and leaked. Firemen inhaled fumes and smoke for 15-30 min before donning breathing apparatuses that were worn intermittently. The gloves of some firemen were soaked, whereas some did not wear gloves at all; others had skin contact during cleanup of the fire. No one was overcome or felt weak after exposure. All 14 men noted symptoms beginning $2 \mathrm{~d}$ to 3 mo after the fire, which included extreme fatigue $(\mathrm{N}=8)$, headache $(\mathrm{N}=7)$, muscle weakness $(\mathrm{N}=9)$, and aching joints $(\mathrm{N}=5)$, but none had arthritis. Eight had memory loss, and 6 noted an impaired ability to concentrate. In the ensuing weeks, several experienced irritability, insomnia, impaired balance, and hypertension; I person lost $10 \mathrm{~kg}$. In August 1987, neurobehavioral, medical, and biochemical studies were conducted. After these studies, they underwent 2-3 wk of a detoxification regimen, which included a regulated diet, and two sessions daily of exercise and heat stress (sauna) during which their medical and blood biochemical status was monitored closely. In November 1987-following the detoxification trial period-neurobehavioral testing was repeated. Also in November a matched comparison group of 14 firemen were studied who had not been in the February fire. In this paper we compare the neurobehavioral function of the exposed group before and after detoxification.

\section{Methods}

The 14 male firemen had fought the fire in the transformer room and were exposed to PCBs and byproducts during control (knock-down) overhaul and cleanup of the fire and equipment. The assistant fire chief assigned a case-matched control group. This control group was selected from firemen who resided in the same city and who were with companies not engaged in the fire in question. These men were matched for age, race, and years as a fireman.

The neurobehavioral test battery was slightly modified from that used in studies of histology technicians exposed to formaldehyde and solvents. ${ }^{9}$ Therefore, instead of doing the sharpened Romberg, body balance was measured using a sound emitter mounted on the head to register its position and speed of movement. A nonverbal, nonarithmetic measure of aptitude or intelligence was added, Culture Fair $2 \mathrm{~A}$ and $2 \mathrm{~B},{ }^{10,11}$ each battery consisting of four sets of picture puzzles. Also, the Profile of Mood States (POMS) ${ }^{12}$ was given to measure affective disorders. Culture Fair and POMS were administered to groups of four to eight firemen. Other testing at both sessions was done individually by trained and experienced physicians or technicians.

Visual two choice reaction time (CRT) was defined as the time from appearance of an A or S on a video screen of a personal computer to its cancellation by pressing a key with the index finger of the dominant hand. The stimulus was generated by a pseudo-random numbers array by the computer with intervals between 2 and $6 \mathrm{~s}$. The median of 7 correct responses to stimuli following 13 responses for learned was recorded as choice reaction time. ${ }^{13}$ Average CRT for adults age 43 y is $600 \pm 88 \mathrm{~ms} .{ }^{9}$

Body balance while standing erect with feet together for I min tests the vestibular apparatus, cerebellar function, proprioception, and motor function. Deviations from standing postural fixation were recorded using a tone signal-generating stylus mounted on the head and two microphones fixed at $10-15 \mathrm{~cm}$ from the head. This Doppler system records the sequential positions of the head and stores this path in a computer (Atari 520). The moments of the path 
were plotted on the $\mathrm{X}$ and $\mathrm{Y}$ axes for a subject standing for $60 \mathrm{~s}$ with eyes open and then for 60 $\mathrm{s}$ with eyes closed. Results were expressed as mean radius in $\mathrm{cm}$ of the sway path. ${ }^{14}$

Memory was measured by administering three tests from Wechsler's memory scale. ${ }^{15}$ The first consisted of immediate recall of two brief stories, read one at a time. Visual memory was then tested by having the subject draw three diagrams immediately after each was presented for $10 \mathrm{~s}$. The third test-digit span measured immediate recall of sequences of numbers read forward and recalled forward and of numbers read forward but recalled backward.

Two-dimensional block designs were assembled from four and then nine blocks to test visual perception and problem solving. This test, from the Wechsler Adult Intelligence Scale ${ }^{16}$ bases scores on the time needed to complete each design.

Embedded figures tests discrimination in recognizing 10 simple objects in each of 4 over-printed arrays. The number of objects recognized in each plate in $30 \mathrm{~s}$ and $60 \mathrm{~s}$ was scored. Poor performance has been correlated with mild lead intoxication in battery workers and with exposure to solvents in painters. ${ }^{20}$

Culture Fair is a nonverbal, nonarithmetic intelligence scale consisting of four subtests selecting designs for similarity, differences, completion, and recognition of relations. ${ }^{10,11}$ This scale, alone or combined with block design, and verbal and visual memory provides an estimate of intelligence.

Four tests that measure dexterity, coordination, decision making, and peripheral sensation from the HalstedReitan battery ${ }^{17}$ are administered in sequence after the memory and block design tests. The time required to place 25 keyed pegs in a slotted pegboard, one at a time, as fast as possible with dominant and nondominant hands, measures dexterity and eye-hand coordination. ${ }^{18}$

Perceptual motor speed (coordination) is measured in trail making A: the time needed to connect 25 numbered dots in consecutive order by a continuous pencil line is measured. Normal subjects require 25$30 \mathrm{~s}$. Trail making B adds decision making, for numbers and letters must be connected alternately in ascending order 1-A, 2-B, up to 13. For normal subjects, this requires 60-65 s, i.e., twice the time of trails A. ${ }^{19}$

Finger writing consists of recognizing numbers 3-6 written in random order four times on each finger pad of both hands, while the subject's eyes are closed. Failures are recorded and reflect impaired peripheral sensory awareness or inability to recognize peripheral stimuli. ${ }^{17}$

The POMS records tension/anxiety, anger, depression, vigor, fatigue, and confusion during the preceding week by having subjects rate 65 adjectives descriptive of feeling on a 0-4 intensity scale. Scores are added in each of the 6 categories. For the POMS score, the vigor score is subtracted from the sum of the other 5 scores.

Following initial evaluation and neurobehavioral testing, the 14 exposed firemen underwent a 2-3 wk detoxification program ${ }^{21,22}$ that included a supervised diet, daily exercise, and sauna. The diet included a polyunsaturated oil supplement and gradually increasing doses of niacin. Aerobic exercise for 30-60 min twice a day was alternated with sauna to $155^{\prime} \mathrm{F}\left(68.3^{\prime} \mathrm{C}\right)$ to increase fat metabolism and sweating. Firemen were monitored with physical and neurological examinations and serum and urine chemical assays with replacement of minerals lost by sweating. The 14 men completed the program, and 14 of 14 completed all of the repeated neurobehavioral testing $6 \mathrm{wk}$ later.

Data were entered into a microcomputer (HewlettPackard 9816), and the basic statistical library was used to compare mean scores of component tests, calculate confidence intervals, and do $t$ tests. A correlation matrix and linear correlations were done with this statistical library. A difference of $\mathrm{p}<.05$ was adopted for significance.

\section{Results}

Initial testing showed that firemen exposed to PCBs had poorer neurobehavioral function than did the case-matched firemen unexposed at this incident (referents) (Table 1). Significant differences were measured for verbal memory, visual memory, digits backward, block design, trails A and B, Culture Fair, and choice reaction time. There were no differences between exposed and referents in dexterity measured 
by pegboard, in balance, in digits forward, and in the numbers of figures recognized during finger writing (Table 1). Exposed firemen showed poorer recall of both Wechsler stories and of the visual memory diagrams, compared with unexposed referents. Also, their ability to repeat digits backward was reduced relative to the unexposed firemen.

\begin{tabular}{|c|c|c|c|}
\hline & $\begin{array}{l}\text { Controls } \\
(N=14) \\
(X \pm S D)\end{array}$ & $\begin{array}{c}\text { Initial for } \\
14 \text { exposed } \\
(X \pm S D)\end{array}$ & $\begin{array}{c}\text { Final for } \\
13 \text { exposed } \\
(X \pm S D)\end{array}$ \\
\hline Age (y) & $34.9 \pm 8.5$ & $33.6 \pm 8.8$ & \\
\hline Years as fireman & $14.1 \pm 7.7$ & $12.7 \pm 8.2$ & \\
\hline Story 1 & $13.6 \pm 2.8$ & $10.2 \pm 4.0^{*}$ & $13.1 \pm 3.95 \mathrm{t}$ \\
\hline Story 2 & $9.6 \pm 4.1$ & $7.8 \pm 3.6^{*}$ & $10.6 \pm 4.3 \dagger$ \\
\hline Pictures & $11.7 \pm 2.9$ & $9.5 \pm 3.2 *$ & $12.0 \pm 1.9 \dagger$ \\
\hline Digits forward & $6.9 \pm 1.4$ & $6.7 \pm 0.9$ & $6.5 \pm 1.1$ \\
\hline Digits backward & $5.1 \pm 1.2$ & $4.0 \pm 1.4^{*}$ & $4.2 \pm 1.4$ \\
\hline Block design score & $31.9 \pm 6.0$ & $30.0 \pm 5.9^{*}$ & $34.2 \pm 9.4 \dagger$ \\
\hline Pegboard dominant & $70.4 \pm 6.6$ & $70.9 \pm 8.8$ & $70.8 \pm 14.5$ \\
\hline Trails A & $27.8 \pm 6.5$ & $36.3 \pm 14.1^{*}$ & $30.4 \pm 12.2$ \\
\hline Trails B & $66.4 \pm 17.3$ & $84.3 \pm 26.7 *$ & $66.5+18.5+$ \\
\hline Finger writing rt. & $1.7 \pm 1.5$ & $2.6 \pm 1.9$ & $3.0 \pm 2.6$ \\
\hline Finger writing lft. & $2.3 \pm 1.9$ & $2.5 \pm 2.1$ & $2.4 \pm 1.9$ \\
\hline Embedded figures & $31.4 \pm 5.0$ & $29.3 \pm 4.8$ & $33.5 \pm 4.9 \dagger$ \\
\hline Culture Fair & $63.4 \pm 6.3$ & $59.8 \pm 9.1^{*}$ & $63.1 \pm 10.1$ \\
\hline Choice reaction time (ms) & $572.0 \pm 81.0$ & $646.0 \pm 150.0^{*}$ & $674.0 \pm 148.0$ \\
\hline Balance & & & \\
\hline Mean dist. eyes open $(\mathrm{cm})$ & $1.30 \pm .33$ & $1.30 \pm 36$ & $1.68 \pm .84 \dagger$ \\
\hline Mean dist. eyes closed $(\mathrm{cm})$ & $1.55 \pm .86$ & $1.47 \pm 1.02$ & $1.68 \pm .84$ \\
\hline POMS 1 Tension & $11.2 \pm 7.2$ & $13.9 \pm 8.0$ & $14.3 \pm 12.3$ \\
\hline POMS 2 Anger & $9.5 \pm 8.1$ & $12.6 \pm 11.3 *$ & $11.6 \pm 7.0$ \\
\hline POMS 3 Depression & $8.7 \pm 9.4$ & $13.8 \pm 11.9 *$ & $14.9 \pm 13.3$ \\
\hline POMS 4 Vigor & $19.1 \pm 3.1$ & $10.3 \pm 7.4^{*}$ & $12.2 \pm 8.0$ \\
\hline POMS 5 Fatigue & $5.9 \pm v 3.3$ & $14.7 \pm 7.4^{*}$ & $11.7 \pm 5.7$ \\
\hline POMS 6 Confusion & $6.4 \pm 3.3$ & $8.3 \pm 5.3$ & $11.7 \pm 5.7$ \\
\hline
\end{tabular}

The cognitive performance of exposed firemen was significantly lower on Culture Fair, with a group mean score of 59.8 vs. 63.4 in referents, and in assembly of block designs, with a mean score of 30.0 vs. 33.0 in controls. Perceptual motor speed and visual spatial performance as measured for trail making A and $\mathrm{B}$ were both significantly prolonged.

Median choice reaction time was $75 \mathrm{~ms}$ longer in exposed firemen than in referents, which is a significant difference. There was no difference in the performance of exposed and unexposed firemen on balance, measured by mean radius of the path moved when eyes were open $(1.30 \mathrm{~cm})$ and when eyes were closed $(1.47 \mathrm{~cm})$.

The POMS showed a significantly lower score for vigor and significantly higher scores for depression, anger, and fatigue in exposed firemen, compared with referents. Tension and confusion scores were also higher, but these differences were not significant. These individual affective disorder scores and POMS score were arrayed with scores on neurobehavioral tests and blood and fat PCB levels in a correlation matrix, and the associations were tested by linear regression. Total POMS score and individual scores for confusion, anger, fatigue, and depression were significantly correlated, and these were correlated with 
balance distance, eyes closed. However, there were no significant correlations with other neurobehavioral tests, including memory, cognitive performance, intelligence, dexterity, and eye-hand coordination, in choice reaction time. The correlation of POMS score with balance radius, eyes closed, probably occurred by chance, and I of 36 items was compared in the correlation matrix. Serum PCB as Arochlor 1248 ranged from 1.9-15 ppb (median of $6.0 \mathrm{ppb}$ ), and body fat content was from 0.15-1.81 ppm (median of $0.7 \mathrm{ppm}$ ). Serum PCB and body content of PCBs were not correlated significantly, nor was either correlated significantly with any neurobehavioral test. Because PCB values varied widely, but were within the ranges described for environmentally exposed human populations, ${ }^{23,24}$ it did not seem useful to repeat them after the detoxification experiment.

After a 3-mo interval, during which the exposed firemen participated in a detoxification program, retesting showed that memory tests were significantly improved, compared with the initial tests for both stories and visual reproduction. Mean scores for cognitive functions measured by block designs and embedded figures also improved significantly, and the improvement in Culture Fair was just short of significant. Trails B, a cognitive and motor performance test, also improved significantly. Only one measure, balance performance (eyes open), worsened significantly and moved against the trend. Choice reaction time, pegboard performance, and recall of digits forward and backward did no change significantly across the interval. Small change in POMS scores on repeat testing were not significant.

\section{Discussion}

These firemen, exposed to heated and exploded PCBS, presumably containing dibenzofurans and other products, had experienced symptoms typical of PCB exposure, ${ }^{8,25}$ including memory loss, headache, irritability, impaired balance, mental slowing, joint aching, muscular weakness, and fatigue.

Neurobehavioral testing of the 14 exposed firemen approximately 6 mo after the fire had demonstrated impairment of verbal memory, visual memory, and recall of digits backward; and reduced cognitive ability to recognize similarity, distinguish differences, complete designs, and transfer relationships in Culture Fair, block design, and embedded figures. Perceptual motor speed in choice reaction time and trail making A and discriminatory motor function on trail making $\mathrm{B}$ had been impaired.

Eight wk after a 2-3 wk detoxification program, memory and cognition in the 14 exposed firemen, as measured by scores on verbal and visual memory, block design, trails B, and embedded figures, had improved significantly and almost matched the scores of the referent firemen. In contrast, balance with eyes open had worsened, as had choice reaction time. Recall of digits and dexterity were unchanged.

In this study, PCB-exposed firemen showed cerebral dysfunction for short-term memory, interpretation of designs, spatial relation integration, decisionmaking, and coordination. The 2-3 wk intensive detoxification program was associated with improved cognitive function and memory. But body balance and choice reaction time did not improve and, in fact, tended to be more abnormal on the second testing in November 1987. As well, performance on the grooved pegboard did not change, nor did peripheral sensation improve. Thus, the changes across the detoxification interval were selective. There were minimal changes in affective status between the testing sessions, which suggests that although some neurophysiological functions improved, the subjects' perception of distress did not improve.

There is concern that memory and cognitive function may improve on repeat testing because of familiarity, practice, motivation, or learning. It would have been helpful to have retested the referent firemen across a 3-mo interval, but this was not possible. However, in a group of individuals followed for formaldehyde exposure, no changes in scores with this test battery were seen 3 mo apart and from year to year. ${ }^{26}$ If there was improvement from test familiarity or small "learning effects," these were evidently offset by age-associated reductions in performance. Thus, we consider it unlikely that the improved function recorded across the test-retest interval in firemen reflects test familiarity or learning. Also, there had been sufficient trials with reaction time and balance testing to produce reproducible resuits on each occasion. $^{13,14}$

Another concern is whether affective disorders adversely affect neurobehavioral test scores as does posttraumatic stress. To examine this problem, we used a correlation matrix to test the composite POMS 
score and individual scores for tension, depression, anger, vigor, fatigue, and confusion for association with scores on individual neurobehavioral tests. Because only one test-balance with eyes closed-was significantly correlated with POMS score in a 36-member matrix, we concluded that in these firemen, affective disorders were not causing neurobehavioral dysfunction.

An ideal experiment would have matched exposed and referent firemen and tested them before the incident, and testing would have been repeated after exposure and again after detoxification. Our opportunities for observation were limited and fell short of such an experimental design. Opportunities were most restricted by our learning of this PCB incident several months after it occurred. Thus, this preliminary report does not presume to answer all questions about the neurotoxicity of PCBs and firegenerated products, but it is intended to alert others to the possibility of chronic neurobehavioral impairment so that the problem can be investigated.

The question of whether the detoxification program was effective was not answered conclusively. Spontaneous improvement of this degree between tests seems unlikely because of the protracted symptoms and self-perceived continued impairment prior to our initial neurobehavioral testing. However, because memory and cognitive function may be influenced by prior testing, we suggest caution in attributing the firemen's improvement exclusively to detoxification.

If these observations are verified, it raises one further problem: firemen who fought fires for 2-25 y probably had other exposures to neurotoxic chemicals; therefore, they may not be an adequate referent group. However, impairment of the referent group would be expected to diminish the differences between the groups, not increase them. If the referent group were impaired, it should raise concern about cumulative neuropsychological and neurophysiological impairment of firemen from repeated brief exposures to high doses of neurotoxic chemicals. Further studies are needed to sort the effects of aging coupled with occasional high chemical exposures from aging alone in impairing neurobehavioral function in firemen.

The failure to find a close response relationship between Arochlor 1248 and impairment on any neurobehavioral tests, although disappointing, suggests that a wider spectrum of PCBs and products must be measured in future studies. This is because polychlorinated dibenzofurans are 1 00-1 0000 times more toxic in animal experiments than PCBS, which have a relatively low toxicity. ${ }^{27-29}$ It is safe to assume that at least part of this difference extends to human subject's neurotoxicity. ${ }^{29}$

Perhaps, as suggested by this study, subjects with neurobehavioral dysfunction from PCBs and PCDFs can be aided by diet, exercise, and sauna-induced sweating aimed at mobilization and excretion of PCBs and their by-products before the exact chemical responsible or its mechanism of action on the central nervous system is understood.

$$
* * * * * * * *
$$

Submitted for publication February 4, 1989; revised; accepted for publication May 14, 1989.

Requests for reprints should be sent to: Kaye H. Kilburn, M.D., University of Southern California School of Medicine, Environmental Sciences Laboratory, 2025 Zonal Avenue, MHR 913, Los Angeles, CA 90033.

$* * * * * * * * * *$

\section{References}

1. Buser HR. Formation, occurrence and analysis of polychlorinated dibenzofurans dioxins and related compounds. Environ Health Persp 1985;60:259-67.

2. Hutzinger 0, Choudhry GG, Chittim BG, Johnston LE. Formation of polychlorinated dibenzofurans and dioxins during combustion, electrical equipment fires and PCB incineration. Environ Health Persp 1985;60:3-9. 
3. Fitzgerald EF, Standfast SJ, Youngblood LG, Melius JM, Janerich DT. Assessing the health effects of potential exposure to PCBS, dioxins, and furans from electrical transformer fires: the Binghamton state office building medical surveillance program. Arch Environ Health 1986;41:368-76.

4. Kuraksune M, Yoshemura T, Matsuzaka J, Yamaguchi A. Epidemiologic study on Yusho, a poisoning caused by ingestion of rice oil contaminated with a commercial brand of polychlorinated biphenyls. Environ Health Persp 1972;1:119-28.

5. Poland AP, Smith D. A health survey of workers at a 2, 4-D and 2, 4, 5-T plant. Arch Environ Health 1971; $22: 316-27$.

6. Huff JE, Moore JA, Saracci R, Tomatis L. Long-term hazards of polychlorinated dibenzodioxins and polychlorinated dibenzofurans. Environ Health Persp 1980;36:221-40.

7. Murai Y, Kuroiwa Y. Peripheral neuropathy in chlorobiphenyl poisoning. Neurology 1971;21:1173-76.

8. Chen RC, Tang SY, Miyata H, Kashimoto T, Chang YC, Chang Kj, Tung TC. Polychlorinated biphenyl poisoning: correlation of sensory and motor nerve conduction, neurologic symptoms, and blood levels of polychlorinated biphenyls, quaterphenyls, and dibenzofurans. Environ Research 1985; 37:340-48.

9. Kilburn KH, Warshaw R, Thornton )C. Formaldehyde impairs memory, equilibrium, and dexterity in histology technicians: effects which persist for days after exposure. Arch Environ Health 1987; 42:117-120.

10. Cattell RB. Classical and standing score IQ standardization of the IPAT: culture free intelligence scale 2. J Consulting Psych 1951; 15:154-59.

11. Cattell RB, Feingold SN, Sarason SB. A culture free intelligence test 11 evaluation of cultural influences on test performance. j Educational Psychol 1941;32:81-100.

12. Profile of Mood States. San Diego, CA: Educational and Industrial Testing Service, 1971/1981.

13. Miller JA, Cohen GS, Warshaw R, Thornton JC, Kilburn KH. Comparison of choice (CRT) and simple reaction times (SRT) in laboratory technicians. Am J Ind Med 1989;15:687-97.

14. Warshaw R, Kilburn KH. Human body balance: a computerized assay of sway (in preparation).

15. Wechsler D. A standardized memory scale for clinical use. J Psychol 1945;19:87-95.

16. Wechsler D. Adult Intelligence Scale Manual (revised). New

York: The Psychological Corporation, 1971.

17. Reitan RM. A research program on the psychological effects of brain lesions in human beings. in: Ellis NR, ed. International review of research in mental retardation. New York: Academic Press, 1966.

18. Costa LD, Vaughan HG, Levita E, Farber N. The Purdue pegboard as a predictor of the presence and laterality of cerebral lesions. Consul Clin Psychol 1963;27:133-147.

19. Reitan RM. Validity of the trail-making test as an indicator of organic brain damage. Percept Motor Skills 1958;8:271-76.

20. Valciukas JA, Sin r RM. The embedded figures test in epidemiological studies of environmental neurotoxic agents. Report to the National Institute of Environmental Health Sciences, 1979.

21. Root DE, Anderson J. Reducing toxic body burdens advancing an innovation technique. Occup Health Safety News Digest 2:1986.

22. Schnare DW, Ben M, Shields MG. Body burden reductions of PCBs, PBBs and chlorinated pesticides in human subjects. Ambio 1984;13:378-80.

23. Wasserman M, Wasserman D, Cucos S, Miller Hj. World PCBs map: storage and effects in man and his biologic environment in the 1970's. Ann NY Acad Sci 1979; 320:69-124.

24. WHO Regional Office for Europe PCBs, PCDDs and PCDFs. Prevention and control of accidental and environmental exposures. Environmental Health Series No. 12, 1987; 205-07.

25. Fischbein A, Wolff MS, Lilis MR, Thornton J, Selikoff Ij. Clinical findings among PCB-exposed capacitor manufacturing workers. Ann NY Acad Sci 1979;320:704-14.

26. Kilburn KH, Warshaw R, Thornton JC. A prospective study of neurobehavioral effects of occupational formaldehyde and solvent exposure in histology technicians (in preparation).

27. Poland A. Studies on the mechanism of action of the chlorinated dibenzo-p-dioxins and related compounds. Ann NY Acad Sci 1979;320:214-29.

28. Neal RA. Mechanisms of the biological effects of PCBs, polychlorinated dibenzofurans in experimental animals. Environ Health Persp 1985;60:41-46.

29. Kashimoto T, Miyata H, Kunita S, Tung TC, Hsu ST, Chang Ki, Tang SY, Ohi G, Nakagawa J, Yamamoto SK. Role of polychlorinated dibenzofuran in Yusho (PCB poisoning). Arch Environ Health 1981; 36:321-26. 